\title{
Single nucleotide recognition using a probes-on-carrier DNA chip
}

$\overline{\text { Saifullah', Satoshi Fuke }^{\ddagger, 1}, \text { Hiroshi Nagasawa }{ }^{3} \text { \& Toshifumi Tsukahara*,1,2 }}$

\section{ABSTRACT}

Following the sequencing of the human genome, SNP analysis of individual patients has become essential for achieving the best drug response and ensuring optimal care. In this study, we developed a costeffective probes-on-carrier DNA chip for the detection of SNPs. Our chips harbored three different probes against the TP53 gene, and were capable of detecting wild-type TP53 and SNPs such as rs121912651 and rs11540652. Four cell lines were used to validate the specificity of probe hybridization. Strong fluorescence intensity was observed in hybridized spots based on hybridization for perfect base pairing between complementary strands, whereas significantly lower fluorescence $(p<0.05)$ was observed in nonhybridized spots. These hybridization results indicate that the probes-on-carrier chip is suitable for SNP genotyping.

\section{KEYWORDS}

genotyping $\cdot$ hybridization $\cdot$ microarray $\cdot$ probes-on-carrier chip $\cdot \mathrm{SNP}$

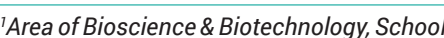
of Materials Science, Japan Advanced Institute of Science \& Technology (JAIST), 1-1 Asahidai, Nomi, Ishikawa 923-1292, Japan; ${ }^{2}$ Division of Transdisciplinary Science, Japan Advanced Institute of Science \& Technology, 1-1 Asahidai, Nomi city, Ishikawa 923-1292, Japan; ${ }^{3}$ Kankyou Resilience, 79-7 Tokiwadai, Hodogaya, Yokohama, 240-0067, Japan; *Author for correspondence: tukahara@ jaist.ac.jp; ${ }^{\ddagger}$ Current address: Unit Research/ Neuroscience Sohyaku, Innovative Research Division, Mitsubishi Tanabe Pharma Corporation, 1000, Kamoshida-Cho, Aoba-Ku, Yokohama 227-0033, Japan

BioTechniques 66: 73-78 (February 2019) 10.2144/btn-2018-0088

\section{LAY ABSTRACT}

Humans differ from each other both genetically and phenotypically. Due to this variation, a given drug will not necessarily be the best choice for all patients. Accordingly, genetic variation should be assessed before medications are selected. DNA microarrays have become popular for the determination of variations (e.g., SNPs), but most of these platforms are expensive, costing up to $\$ 1000$ per chip. Consequently, it is costprohibitive to use these chips for individual clinical tests. We developed an economical DNA chip that can evaluate more than 1000 SNPs at a cost of less than $\$ 10$ per chip.

\section{METHOD SUMMARY}

To implement a probes-on-carrier DNA chip as a genotyping tool, we used a fragment of the TP53 gene from four different cell lines (target DNA). To confirm single-base specificity, we hybridized the chip-containing probes with labeled target DNA.

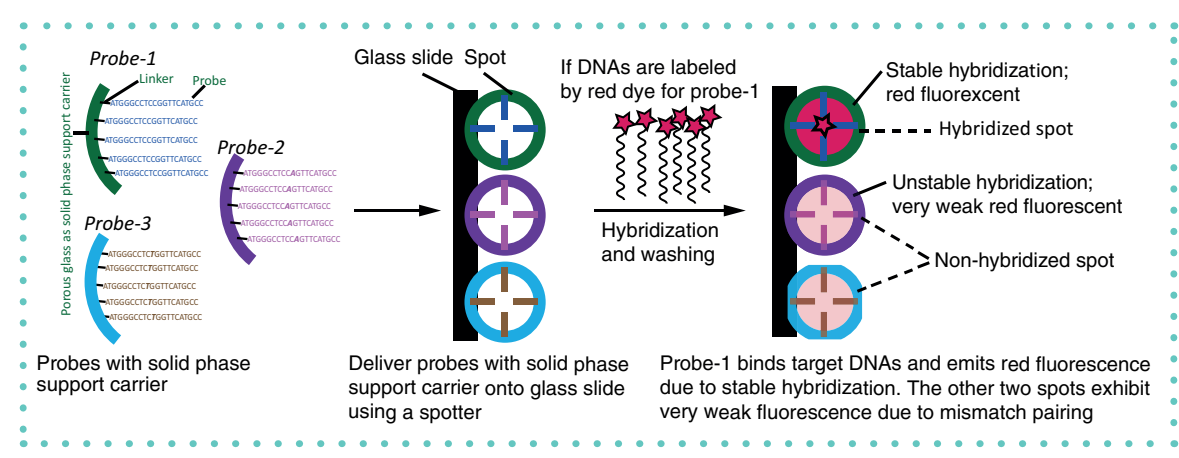

Three probes with solid-phase support carrier were spotted on a glass slide to make a probeson-carrier DNA chip. These probes were designed for the detection of TP53wt and two SNPs: rs121912651 and rs11540652. If labeled complementary DNAs for probe-1 are employed on the chip, only probe-1 binds target DNAs and emits relatively strong fluorescence.

\section{INTRODUCTION}

Genes related to drug absorption, distribution, metabolism and elimination (ADME) are polymorphic, causing the efficacy and toxicity of drugs to vary among individuals [1,2]. Accordingly, assessment of genetic variation, including SNPs, is a prerequisite for achieving the best drug response and highest safety margins, thereby improving patient care as a component of personalized medicine. In addition to pharmacogenomics, SNPs also play important roles in studies of oncology [3] and inheritance of monogenic diseases of development [4], and also as biomarkers in population genetics [5]. SNPs, which are single-nucleotide variations at specific loci, are the most frequent type of subtle genetic variations in the human genome.
Allele-specific probe hybridization on DNA microarray platforms is a modern and powerful technique for high-throughput genotyping. Oligo-DNA array chips are produced using semiconductor photolithography, bead conjugation or ink-jet technology [6]. However, these technologies are very expensive and time-consuming and require sophisticated cleanroom facilities. In addition, bubble-jet technology requires that the probes undergo 5'-modification and the addition of extra cross-linkers or functional residues so that they can be attached to the solid substrate [7]. These limitations present a significant obstacle to the wide adoption of microarray chips in diagnostic contexts, especially in developing countries where cutting-edge laboratories are rare.

We report a cost-effective probes-oncarrier DNA chip by immobilization of 


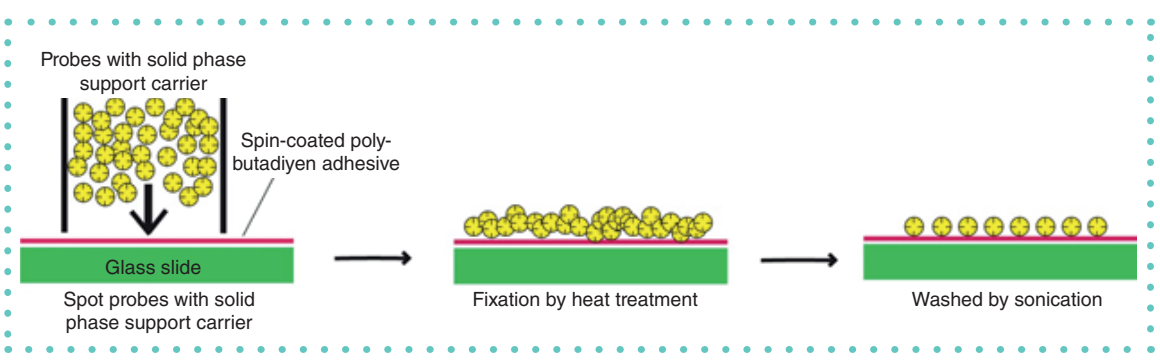

Figure 1. Preparation of a probes-on-carrier DNA chip. Probes with a solid-phase support carrier were immobilized onto a glass slide with the help of an adhesive by using the spotter machine. Spots were fixed and excessive spots were removed by heat treatment and sonication method, respectively.

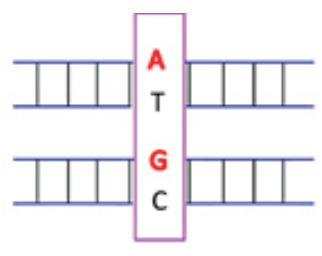

$\operatorname{SNP}(\mathrm{A} / \mathrm{G})($ e.g., rs11540652)
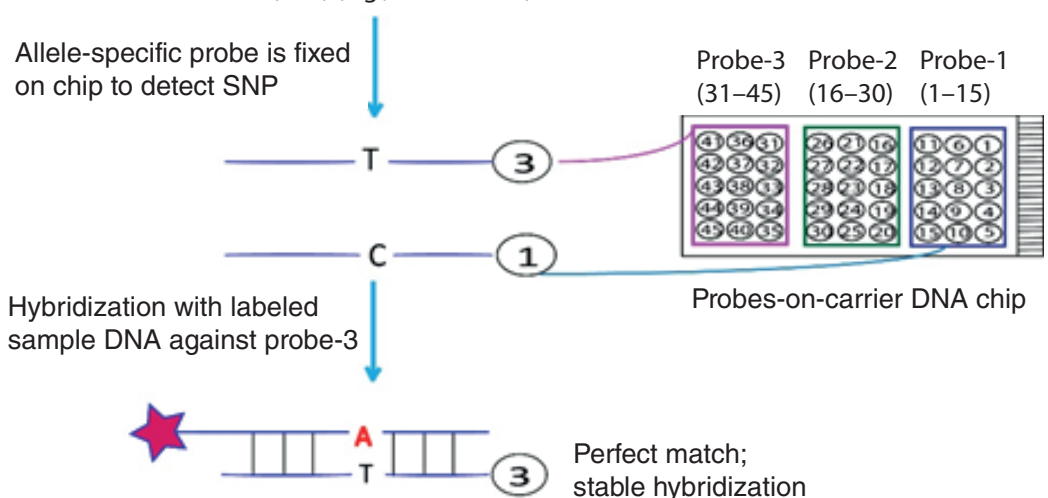

Perfect match; stable hybridization

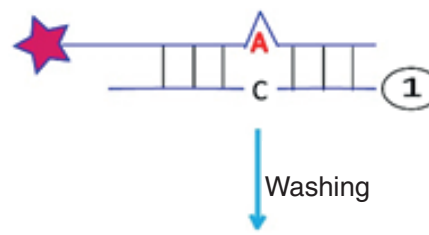

Mismatch; unstable hybridization
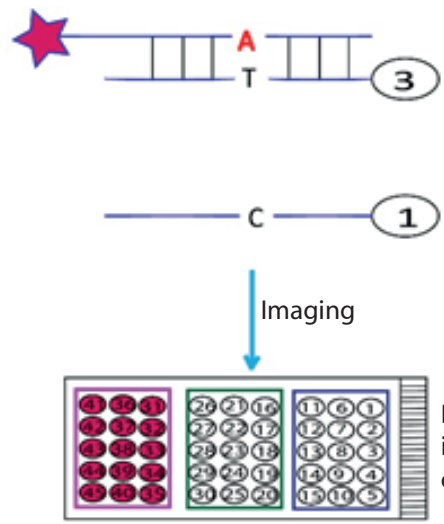

Red fluorescent wells in 35-45 of probe-3 indicates the probe hybridized with Allele A containing complementary labeled sample DNA

Probe-3 Probe-2 Probe-1

Figure 2. Working mechanism of the chip. Here, rs 11540652 is used as an example. The target is an A/G SNP. The C-probe (probe-1) for the $G$ allele and the T-probe (probe-3) for the A allele are immobilized on the chip. Upon hybridization with labeled target DNA from HSC-4 cells, DNAs containing the A allele perfectly matched with probe- 3 and emitted red fluorescence. Probe-1 also hybridized with A allele DNA, but washed out due to unstable hybridization.
cDNA probes on solid-phase Controlled Pore-Glass (DNA-CPG) [8]. Ohkubo et al. (our collaborators) used the DNA-CPG method for SNP detection using the chemical ligation (CL) strategy [9]. Later, they used a modified method of probes-oncarrier called protected DNA probes (PDP) capable of strong hybridization without conventional ammonia treatment [10]. However, modifying probe sequences with specific chemical groups for CL strategy and PDP are problematic for the liberal use of the DNA chip. Besides that, all these techniques used synthesized-oligos to check the performance of CL strategy and PDP method. Therefore, to realize the clinical use of the chip, an application of the probes-on-carrier DNA chip without any chemical modification of probes, for the identification of SNPs in the TP53 gene from direct cell DNAs on a small scale, has been presented here. The probes-on-carrier DNA chip is a platform consisting of a porous microscopic glass slide on which there are immobilized presynthesized oligonucleotides, facilitating identification of singlebase variations in the genome.

\section{MATERIALS \& METHODS Probes-on-carrier DNA chip}

A probes-on-carrier DNA chip is a porous borosilicate glass slide on which presynthesized oligonucleotide probes are placed by a spotter machine, and subsequently chemically immobilized. In this study, probes-on-carrier DNA chips were prepared as previously described [8]. Briefly, three kinds of probes with sequences identical except at one nucleotide (sequences are provided in Figure $4 \mathrm{~A}-\mathrm{C}$ ) were synthesized separately on hexaethyleneglycol linker with porous glass as a solid-phase support carrier by an automatic oligonucleotide synthesizer. These probes were designed for detection of TP53wt and two SNPS, rs121912651 and rs11540652. They were spotted onto a porous glass slide with the aid of spin-coated polybutadiene adhesive. Heat treatment and ultra-sonication were performed to fix the probes and removal of excess probes from the slide, respectively (Figure 1).

\section{Microarray procedure}

The working mechanism of the probes-oncarrier DNA chip is shown in Figure 2. 


\section{Cell culture \& DNA isolation}

HeLa (ID: JCRB9004), Ca9-22 (ID: JCRB0625), HSC-4 (ID: JCRB0624) and PLC (ID: JCRB0406) cells (JCRB Cell Bank, Osaka, Japan) were cultured in Dulbecco's modified Eagle's medium (DMEM, Nacalai Tesque, Inc., Kyoto, Japan) containing $10 \%$ fetal bovine serum (Gibco, Life Technology, Canada). Genomic DNA (gDNA) from the cell lines was isolated separately as described elsewhere [3]

\section{Polymerase chain reaction (PCR)}

PCR was performed in a total reaction volume of $50 \mu$ l on a GeneAmp PCR System 9700 (Applied Biosystems, Singapore). The reaction mixture contained $35.5 \mu \mathrm{l}$ of autoclave MilliQ water, $5 \mu$ l of $10 \times$ Pfu buffer, $5 \mu \mathrm{l}$ of $2 \mathrm{mM}$ dNTPs, $1 \mu \mathrm{l}$ (10 pmol) of each primer, $2 \mu \mathrm{l}$ of genomic DNA, and $0.5 \mu \mathrm{l}$ of Pfu DNA polymerase (Agilent Technologies, CA, USA). The PCR procedure was as follows: predenaturation at $95^{\circ} \mathrm{C}$ for $3 \mathrm{~min}$; followed by 30 cycles of $95^{\circ} \mathrm{C}$ for $30 \mathrm{~s}, 58^{\circ} \mathrm{C}$ for $30 \mathrm{~s}$, and $72^{\circ} \mathrm{C}$ for 1:30 min; and a final extension at $72^{\circ} \mathrm{C}$ for $10 \mathrm{~min}$. The length of amplified DNA fragments was assessed by $1 \%$ agarose gel electrophoresis, followed by purification using the QIAEX ${ }^{\circledR} \mid$ Gel Extraction Kit (QIAGEN, Germany).

\section{Fluorescent dye labeling}

Direct chemical labeling was performed using the Cy5 fluorescent reaction compound, also called the universal linkage system (ULS). Labeling reactions were carried out with the Genomic DNA ULS labeling kit (Agilent Technologies, USA) according to the Agilent 'Oligonucleotide Array-Based CGH for Genomic DNA Analysis' protocol. Briefly, $16.5 \mu \mathrm{l}$ of purified PCR fragments $(0,1,10$ or $100 \mathrm{ng})$ and gDNA (100 ng) from HeLa cells were labeled with Cy5 fluorescent dye. A similar volume of purified PCR fragments (100 ng) from the other cell lines was also labeled. Labeling reactions were carried out in a $20 \mathrm{ul}$ volume at $85^{\circ} \mathrm{C}$ for $30 \mathrm{~min}$, followed by incubation at $4^{\circ} \mathrm{C}$ for $3 \mathrm{~min}$. Nonreacted Cy 5 dye was removed using a KREApure column (Agilent Technologies, USA). Finally, the degree of DNA labeling was measured on a NanoDrop1000 (ThermoFisher, Japan). the chip to identify target-specific nucleotides. For hybridization of amplified DNA, hybridization reaction mixtures contained labeled PCR-DNA $(\sim 20 \mu \mathrm{l})$ and $2 \times$ hybridization buffer $(20 \mu \mathrm{l})$. For hybridization of gDNA, reaction mixtures consisted of human Cot-1 DNA $(12.5 \mu \mathrm{l}), 100 \times$ blocking agent $(1.3 \mu \mathrm{l}), 2 \times$ hybridization buffer $(65 \mu \mathrm{I})$, labeled gDNA $(\sim 20 \mu \mathrm{l})$, and Agilent-CGHBlock $(32.5 \mu \mathrm{l})$. The reaction mixture was spread on the active side of the chip and incubated at $55^{\circ} \mathrm{C}$ overnight in a shaking incubator.

Low salt concentration results in higherstringency washing to remove unbound DNA. Following hybridization, the slides were first immersed in $2 \times$ SSC solution for disassembly, and then in $0.1 \times$ SSC $/ 0.01 \%$ SDS wash solution. For gDNA, wash buffers $A$ and $B$ (Agilent Technologies, USA) were used at room temperature. The chip was then dried by centrifugation at $700 \times \mathrm{g}$ for $5 \mathrm{~min}$ in a $50-\mathrm{ml}$ centrifuge tube. The chip was immediately subjected to scanning for analysis.
Imaging

Fluorescence from hybridized spots on the chip was observed on an image scanner (CRBIO lle, Hitachi Ltd, Japan) with the following settings: channel, 2: photomultiplier tube (PMT) sensitivity, 70-90; focus, $2 \mu \mathrm{m}$; focus resolution, 5 um; laser power, 90-100; gray level adjustment, default. After the chip was mounted on the scanner, the image resolution was adjusted using the Preview option. The chip was then scanned, and a 16-bit gray-scale TIFF file was saved for further analysis.

\section{Data analysis}

Light intensity of each spot was measured by computing integrated density (sum of the values of the pixels in the image) of the TIFF image using the ImageJ software [11]. The standard error of the mean (s.e.m.), Student t-test and ANOVA test were calculated using the data analysis ToolPak in Microsoft Excel version 16. A p-value of 0.05 was considered as a standard for comparing all statistical significant data. 
(A)

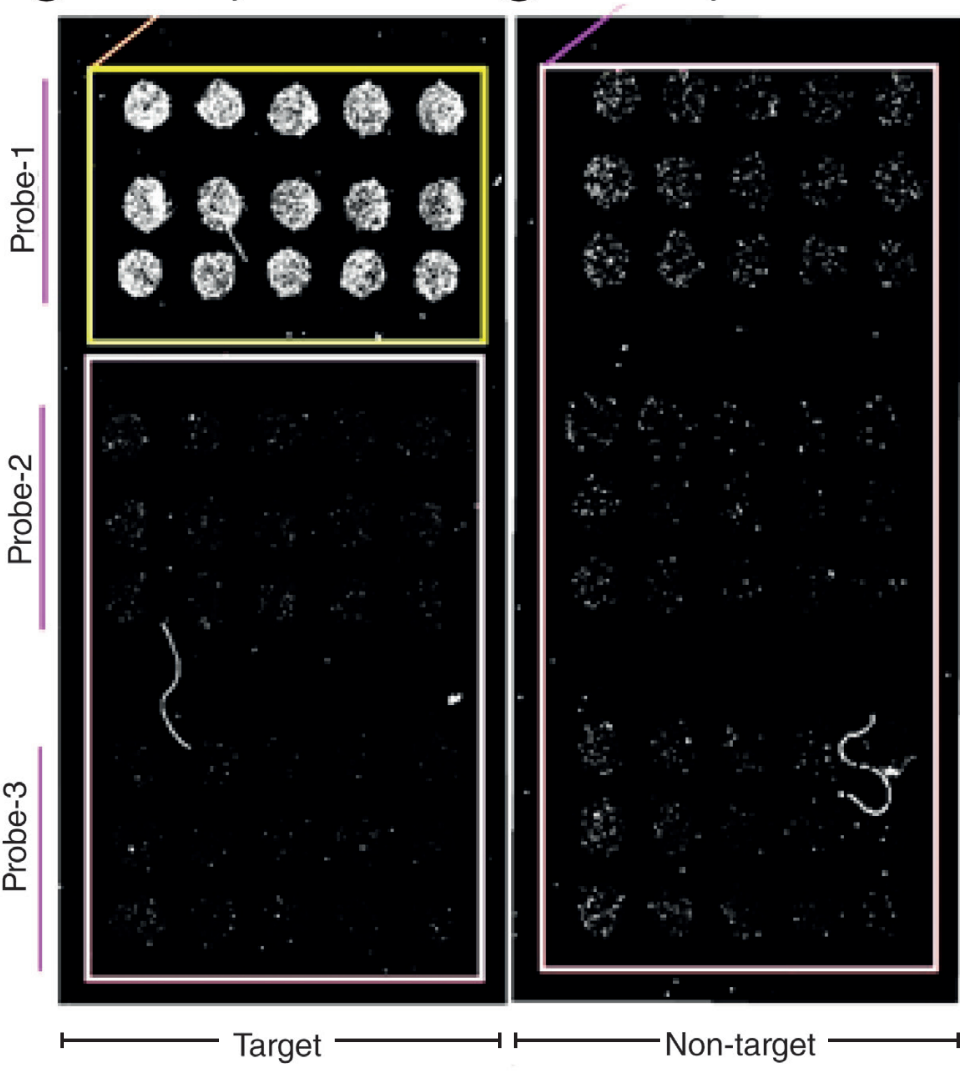

Figure 3. Hybridization versus nonhybridization imaging results. Three sets of probes differing at a single base were fixed on the A and B chips. (A) Complementary DNA to probe-1 (TP53wt) was only applied to the A chip, as a target. Upon hybridization, only the probe-1 spots exhibited fluorescence (yellow rectangular mark; hybridized), whereas the rest of the fluorescence from spots containing probe- 2 and -3 were washed out due to mismatch pairing (pink rectangle; nonhybridized). (B) PCR DNA from PLC cells (SNP accession no. rs28934571) was applied to chip B as a nontarget. Lack of fluorescence indicates no hybridization in any spots (pink rectangle).

\section{- RESULTS \& DISCUSSION}

Determination of DNA concentration PCR results are presented in the supplementary materials and Supplementary Figure S1. PCR-DNA was purified from the agarose gel, and various amounts $(0,1,10$ and $100 \mathrm{ng}$ ) were diluted in water. Labeling and hybridization were performed; the hybridization results demonstrated that a 100 ng DNA concentration is best suited for the hybridization reaction (data not shown). Accordingly, we prepared a positive control using 100 ng DNA from HeLa cells (Figure 4F).

\section{Hybridization results}

Three sets of probes, probe- $1,-2$ and -3 , were fixed on the chip to detect sample DNAs containing TP53wt, rs12191265, and rs11540652, respectively. One type of sample DNA was used on each chip in each hybridization reaction. For instance, PCR-DNA of TP53wt was used to check the hybridization results of probe-1 (Figure $3 \mathrm{~A})$. In the same reaction, PCR-DNA of rs28934571 from PLC cells was used as a nontarget, that is, a negative control (Figure 3B). 15 Probe-1 spots in Figure 3A clearly exhibited stable hybridization (yellow rectangle: strong white color spots), whereas the rest of the spots for probe-2 and -3 were nonhybridized due to mismatch pairing (pink rectangle; much lower fluorescence intensity) (Figure 3).

Hybridization results for six different probes-on-carrier DNA chips are shown in Figure 4. In the graphs, fluorescence hybridization of probes with complementary sample DNA is represented by black patterned boxes, and nonhybridizing spots are represented by white patterned boxes. The results reveal strong fluorescence (fluorescence intensity 107.7) in hybridized spots for obtaining perfect Watson-Crick base pairing between probes and corresponding sample DNAs. On the other hand, in nonhybridized spots, the single mismatch between the complementary strands resulted in unstable hybridization (fluorescence intensity 43.6), and fluorescence was removed upon washing (Figure $4 A-C$ ). For similar reasons, nontarget DNA exhibited no stable hybridization at all (Figure 4D).

Next, we evaluated hybridization of full genomic DNA of HeLa cells against probe-1 oligonucleotide. We observed a similar level of hybridization (Figure 4E). This observation indicates that our chip is suitable for the detection of a single-nucleotide difference within the complex human genome $\left(3 \times 10^{9} \mathrm{bp}\right)$.

We analyzed fluorescence intensity across all samples. For non-hybridized fluorescence, the mean value of all samples was $43.6 \pm 4.0$. For hybridized fluorescence, the mean value was $107.7 \pm 2$.6. To compare the fluorescence intensity between hybridized and nonhybridized spots across all samples, we performed Student's two-tailed t-test with unequal variance on all mean values. The results revealed a significant $(p<0.05)$ difference between hybridized and nonhybridized fluorescence across all spots, suggesting that single-base variation could be detected based on fluorescence. Within hybridized and nonhybridized spots for TP53wt, rs121912651, and rs11540652 target DNAs, no significant difference was detected (one-way ANOVA; $p>0.05$ ).

Gudnason et al. developed a method for immobilization of probes with TC-tag on an unmodified glass slide by using UV-light irradiation for cross-linking [12]. Kim et al. showed that chemical attachment of probes with T20 tag on the aziridine-treated glass slide is better than the UV-light irradiation method for cross-linking to discriminate single nucleotide mismatch [13]. UV-light exposure may exert mutation in probes. Furthermore, even surface modification of the glass slide and covalent linkage of presynthesized probes are critical factors to use the slide as a DNA chip. For that, we used a chemical linker, hexaethyleneglycol, to attach the probes with porous glass as a solid-phase carrier. 
Probes-on-carrier DNA chips offer many advantages over other microarray technologies. First, the chip can distinguish between matched hybrids and single-base mismatch hybrids. Figure $3 A-B$ and Figure $4 A-F$ demonstrate that the probes only bind stably to target DNA samples when they match perfectly; otherwise, they wash out due to unstable hybridization. Second, our chip is cost-effective for personal use. Even though DNA microarrays have become popular, most of these platforms are very expensive, costing thousands of US dollars per chip and making them costprohibitive for use in diagnostic contexts. Here, we describe an economical DNA chip that can perform genotyping of more than 1000 SNPs at a cost of less than USD 10 per chip. Third, chip development is easy and convenient because conventional primer synthesis machines are suitable for probe synthesis. By contrast, existing DNA microarrays require a sophisticated laboratory for photolithography [14] or ink-jet technology [15]. Fourth, like other chip technologies [6], our method is reproducible: as shown by Figure $4 \mathrm{~A}-\mathrm{F}$, no noteworthy difference was detected within hybridized or nonhybridized spots across all chips. Fifth, like presynthesized oligonucleotides, probes-on-carrier DNA chips are very stable and could be stored for a long time, even at room temperature.

However, although stably hybridized spots were clearly distinguishable from unstable hybridized spots, the chip used in this study did exhibit some background noise due to inadequate washing. Our method is not a rapid detection assay, unlike recently emerging microfluidics biochips for identification of avian influenza through DNA hybridization [16] and for the detection of cancer biomarkers or bacteria [17]. However, those assays of biochips were not for SNP detection.

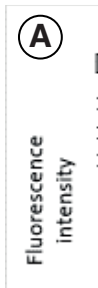

TP53wt (probe-1)

[5'-ATGGGCCTCCGGTTCATGCC-3']

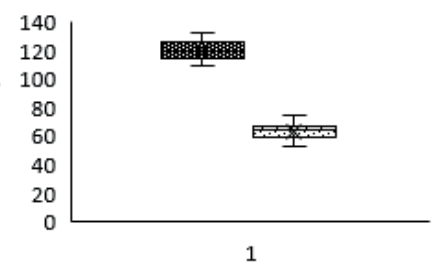

国 Hybridized Non-hybridized

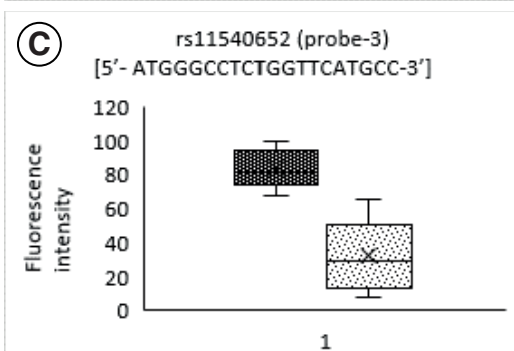

Hybridized Non-hybridized

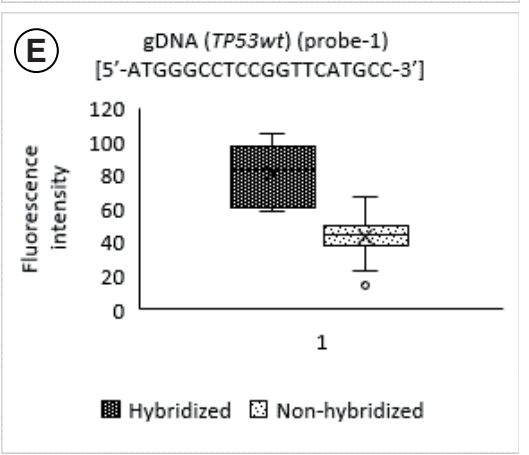

(B)

rs121912651 (probe-2) [5'-ATGGGCCTCCAGTTCATGCC-3']

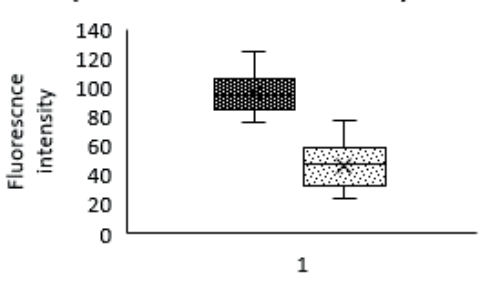

闻 Hybridized Non-hybridized

(D) rs28934571 (Non-target)

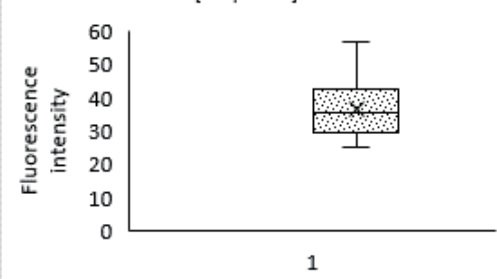

Hybridized Non-hybridized

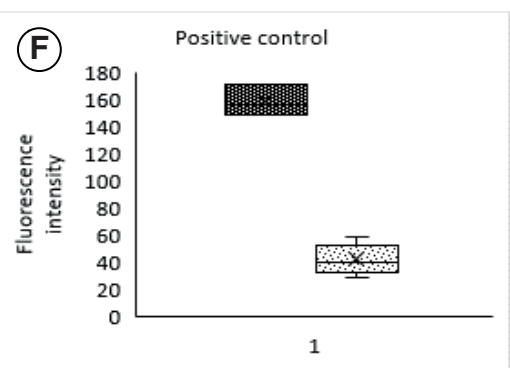

Hybridized Non-hybridized
Figure 4. Results of chip hybridization. 'Hybridized' boxes represent spots exhibiting strong fluorescence, whereas 'nonhybridized' boxes represent weaker fluorescence. (A) PCR-DNA of TP53wt was applied; probe-1 exhibited strong fluorescence and was denoted as hybridized, whereas the other spots exhibited unstable hybridization and were denoted as nonhybridized. (B) PCR-DNA of rs121912651 was applied, and only spots containing probe-2 exhibited hybridization. (C) PCR-DNA of rs 11540652 was applied, and only spots containing probe- 3 exhibited hybridization. (D) PCR-DNA from PLC cells was applied and exhibited no strong fluorescence (nonhybridized), because no complementary probe was on the chip, indicating nontarget DNA. (E) Genomic DNA from HeLa cells was applied to the chip, and only spots containing probe-1 exhibited hybridization. (F) $100 \mathrm{ng}$ PCR-DNA from HeLa cells was used for probe-1 in a different chip as a positive control. Sample number (spots): (A-C): hybridized $=15$, nonhybridized = 30; $(D)$ : nonhybridized $=45 ;(E)$ : ybbridized $=12$, nonhybridized $=24 ;(F)$ : ybridized $=3$, nonhybridized $=6$.

\section{EXECUTIVE SUMMARY}

We demonstrated the use of probes-on-carrier DNA chips as SNP genotyping tools.

- Using this system, we could prepare accurate DNA chips at a cost of less than US\$10.
The probes-on-carrier DNA chip platform has many potential applications, including single-nucleotide detection, diagnosis of cancer risk-associated SNPs, RNA detection in small-scale basic research, and DNA detection for identification of diseaserelated point mutations.

- We hope that this finding will contribute to pharmacoge- nomics, and thereby promote evidence-based drug design, in the near future. 
- Another limitation of this study is that we performed genotyping in a small sample size, as a trial. In the near future, we will use this method to analyze a much larger number of samples.

Currently, probes-on-carrier chips are limited to SNP genotyping, but gene expression analysis could be achieved by designing longer probes (100-150 bp) against genes of interest and introducing large pore size $(\sim 100 \mathrm{~nm})$ in the carrier (i.e., glass slide). We demonstrated previously that it is possible to strictly control pore size in probes-on-carrier DNA chips [8]. In addition, these chips could be used in smallscale basic research. For example, Azad et al. developed an MS2 RNA-editing system for restoration of the G-to-A mutation in gene therapy, and used restriction fragment length polymorphism) and sequencing methods to validate their system [18]. In the near future, we could design a single chip consisting of 1000 different probes, including a target point mutation (e.g., G-to-A), thus enabling detection of 500 different edited target RNAs. Such a chip could be used as an alternative to restriction fragment length polymorphism or sequencing to validate a gene editing system. This approach would be advantageous because DNA chips are economical, convenient and reliable.

\section{AUTHOR CONTRIBUTIONS}

Saifullah, SF and HN performed experiments. TT designed and supervised the study. Saifullah and TT wrote the paper.

\section{FINANCIAL \& COMPETING INTERESTS DISCLOSURE}

This work was supported by Grant-in-Aid for Scientific Research from the Japan Society for the Promotion of Science (26670167 and 17H02204). Saifullah thankfully acknowledges the scholarship from the Ministry of Education, Culture, Sports, Science, and Technology (MEXT), Japan. The authors have no other relevant affiliations or financial involvement with any organization or entity with a financial interest in or financial conflict with the subject matter or materials discussed in the manuscript apart from those disclosed.

No writing assistance was utilized in the production of this manuscript.

\section{DMedNet}

\section{Join our free 3D medical} printing community hub!

Explore - Get exclusive free access to news, features, journal articles and videos

Engage - Share knowledge with a network of international experts from the medical 3D printing and bioprinting community

Enhance - Learn through peer-led webinars encouraging interaction and discussion $\overline{\text { Visit - www.3dmednet.com/ }}$

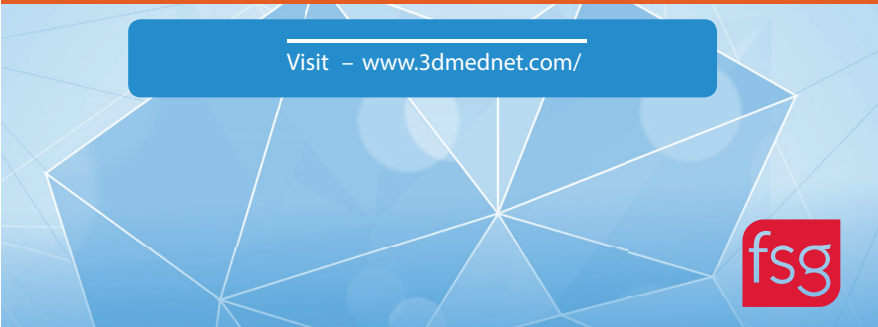

\section{OPEN ACCESS}

This work is licensed under the AttributionNonCommercial-NoDerivatives 4.0 Unported License. To view a copy of this license, visit http://creativecommons.org/licenses/ by-nc-nd/4.0/

\section{SUPPLEMENTARY DATA}

To view the supplementary data that accompany this paper please visit the journal website at: www.future-science. com/doi/suppl/10.2144/btn-2018-0088

\section{REFERENCES}

1. Jittikoon J, Mahasirimongkol S, Charoenyingwattana A et al. Comparison of genetic variation in drug ADME-related genes in Thais with Caucasian, African and Asian HapMap populations. J. Hum. Genet. 61 (2), 119-127 (2016).

2. Evans WE, Relling MV. Pharmacogenomics: translating functional genomics into rational therapeutics. Science 286(5439), 487-491 (1999).

3. Saifullah, Tsukahara T. Genotyping of single nucleotide polymorphisms using the SNP-RFLP method. BiosCi. Trends 12(3), 240-246 (2018).

4. Syvänen A-C. Accessing genetic variation: genotyping single nucleotide polymorphisms. Nat. Rev. Genet. 2(12), 930-942 (2001)

5. Jorde LB, Watkins WS, Bamshad MJ et al. The distribution of human genetic diversity. a comparison of mitochondrial, autosomal, and Y-chromosome data. Am J. Hum. Genet. 66(3), 979-988 (2000)

6. Roger B. Overview of DNA microarrays: types, applications, and their future. Curr. Protoc. Mol. Biol. 101(1) 22.21.21-22.21.11 (2013).

7. Okamoto T, Suzuki T, Yamamoto N. Microarray fabrication with covalent attachment of DNA using Bubble Jet technology. Nat. Biotechnol. 18(4), 438-441 (2000).

8. Tsukahara T, Nagasawa H. Probe-on-carriers for oligonucleotide microarrays (DNA chips). Sci. Technol. Adv. Mater. 5(3), 359-362 (2004)

9. Ohkubo A, Tanaka K, Taguchi H et al. An effective method for the in situ synthesis of DNA-CPG conjugates using chemical ligation technology as tools for SNP analysis. Bioorg. Med. Chem. Lett. 17(21), 5969-5973 (2007)

10. Ohkubo A, Kasuya R, Sakamoto K et al. 'Protected DNA probes' capable of strong hybridization without removal of base protecting groups. Nucleic Acids. Res. 36(6), 1952-1964 (2008)

11. Schneider CA, Rasband WS, Eliceiri KW. NIH Image to Image J: 25 years of image analysis. Nat. Methods 9(7), 671-675 (2012).

12. Gudnason $H$, Dufva M, Duong Bang D, Wolff A. An inexpensive and simple method for thermally stable immobilization of DNA on an unmodified glass surface: UV linking of poly $(T) 10$-poly(C)10-tagged DNA probes. BioTechniques 45(3), 261-271 (2008)

13. Kim E-S, Kim CO, Park JW, Choi KY. Detection of central single-nucleotide mismatches in short duplex DNAs on hyper-branched amine surfaces. Biochip J. 5(2), 137 (2011).

14. Fodor S, Read J, Pirrung M, Stryer L, Lu A, Solas D. Light-directed, spatially addressable parallel chemica synthesis. Science 251(4995), 767-773 (1991)

15. Blanchard AP, Kaiser RJ, Hood LE. High-density oligonucleotide arrays. Biosens. Bioelectron. 11(6), 687-690 (1996)

16. Sun $Y$, Perch-Nielsen I, Dufva $M$ et al. Direct immobilization of DNA probes on non-modified plastics by UV irradiation and integration in microfluidic devices for rapid bioassay. Anal. Bioanal. Chem. 402(2), 741-748 (2012).

17. Xiong $\mathrm{Q}$, Lim CY, Ren J et al. Magnetic nanochain integrated microfluidic biochips. Nat. Commun. 9(1) 1-11 (2018)

18. Azad MTA, Bhakta S, Tsukahara T. Site-directed RNA editing by adenosine deaminase acting on RNA for correction of the genetic code in gene therapy. Gene Ther 24(12), 779-786(2017). 